\title{
Nets of Lines with the Combinatorics of the Square Grid and with Touching Inscribed Conics
}

\author{
Alexander I. Bobenko ${ }^{1}$ - Alexander Y. Fairley ${ }^{1}$ \\ Received: 5 December 2019 / Revised: 29 November 2020 / Accepted: 4 January 2021 / \\ Published online: 10 February 2021 \\ (c) The Author(s) 2021
}

\begin{abstract}
In the projective plane, we consider congruences of straight lines with the combinatorics of the square grid and with all elementary quadrilaterals possessing touching inscribed conics. The inscribed conics of two combinatorially neighbouring quadrilaterals have the same touching point on their common edge-line. We suggest that these nets are a natural projective generalisation of incircular nets. It is shown that these nets are planar Koenigs nets. Moreover, we show that general Koenigs nets are characterised by the existence of a 1-parameter family of touching inscribed conics. It is shown that the lines of any grid of quadrilaterals with touching inscribed conics are tangent to a common conic. These grids can be constructed via polygonal chains that are inscribed in conics. The special case of billiards in conics corresponds to incircular nets.
\end{abstract}

Keywords Discrete differential geometry · Inscribed conics · Inscribed circles · Incidence theorems

Mathematics Subject Classification 51A05 · 53A20 · 52C30 · 37J35

\section{Editor in Charge: János Pach}

Alexander I. Bobenko was partially supported by the DFG Collaborative Research Center TRR 109 "Discretization in Geometry and Dynamics". Alexander Y. Fairley was supported by a Leverhulme Trust Study Abroad Studentship.

Alexander I. Bobenko

bobenko@math.tu-berlin.de

Alexander Y. Fairley

fairley@math.tu-berlin.de

1 Institut für Mathematik, Technische Universität Berlin, Str. des 17. Juni 136, 10623 Berlin, Germany 


\section{Introduction}

The geometry of incircular nets (IC-nets) has recently been discussed in great detail in [1]. IC-nets were introduced by Böhm [7] and they are defined as congruences of straight lines in the plane with the combinatorics of the square grid such that each elementary quadrilateral admits an inscribed circle. IC-nets have a wealth of geometric properties, including the distinctive feature that any IC-net comes with a conic to which the gridlines are tangent. IC-nets are closely related to Poncelet(-Darboux) grids, which were originally introduced by Darboux [10] and further studied in [17,21].

Checkerboard IC-nets constitute a natural generalisation of IC-nets. The gridlines of checkerboard IC-nets have the combinatorics of the square grid but it is only required that every second quadrilateral admits an inscribed circle, namely the "black" (or "white" if the colours are interchanged) quadrilaterals if the quadrilaterals of the net are combinatorially coloured like those of a checkerboard. Checkerboard IC-nets can be consistently oriented so that their lines and circles are in oriented contact. Thus, these nets are naturally treated in terms of Laguerre geometry. In [5] checkerboard ICnets were explicitly integrated in terms of Jacobi elliptic functions. The integration is similar to the case of elliptic billiards [12]. Recently in [4] the corresponding definitions and results were extended to the cases of incircular nets in the 2-sphere and also in the hyperbolic plane by developing the corresponding Laguerre geometries.

In this paper we suggest a purely projective generalisation of IC-nets. Namely, we consider planar congruences of straight lines with the combinatorics of the square grid and with all elementary quadrilaterals possessing touching inscribed conics (see Fig. 12). It is worth mentioning that the lines of the projective grids we introduce correspond not to the lines of IC-nets but to the lines passing through the centres of their circles. We describe their geometry in detail and show, in particular, in Sect. 4.2 that the lines of these grids touch a common conic. A further important property is that planar grids of quadrilaterals with touching inscribed conics are planar Koenigs nets. Koenigs nets are an important example of integrable discrete differential geometry [6]. In Sect. 3.2, we show that the property to possess touching inscribed conics is characteristic for general Koenigs net. This characterisation of Koenigs nets via inscribed conics (Theorem 3.5) was independently discovered by Christian Müller.

Our geometric analysis is based essentially on Theorem 4.2, which is an incidence theorem for five conics and six touching lines, see Fig. 12. The corresponding implications for grids of quadrilaterals with touching inscribed conics are described in Sect. 4. In particular, it is shown that these grids can be constructed via polygonal chains that are inscribed in conics. In Sect. 4.4 it is demonstrated how the special case of billiards in conics can be used to generate incircular nets (see Fig. 1).

\section{Preliminaries}

In this section we present some known results about inscribed conics. Many theorems about quadrilaterals with inscribed conics can be found in, for instance, [9, Chaps. XII, XVI, and XVIII]. Many other theorems about conics can be found in [13]. 


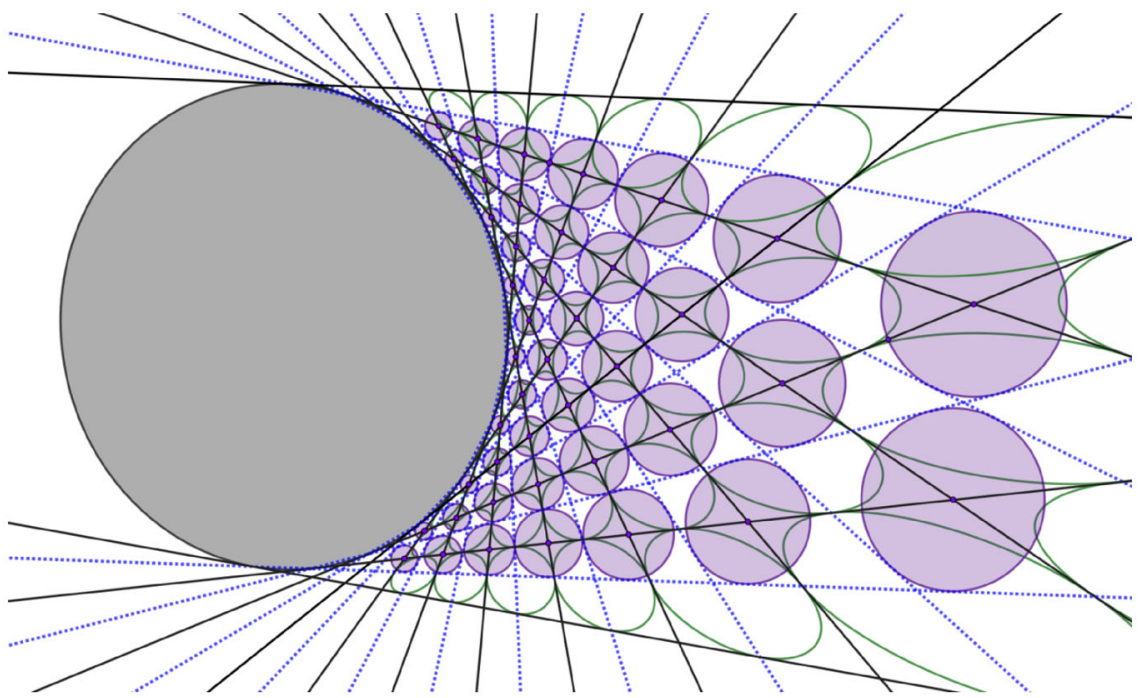

Fig. 1 For any billiard in the grey conic, there is an associated incircular net. More generally, for any polygonal chain inscribed in the grey conic, there is an associated grid of quadrilaterals with touching inscribed conics. The inscribed circles and the touching inscribed conics are in combinatorially dual line grids. The line grid with touching inscribed conics is given by the lines passing through the centres of the circles

In the projective plane $\mathbb{P}^{2}$, any arrangement of lines is called generic if and only if no three of the lines are concurrent. Let $\square\left(v_{1}, v_{2}, v_{3}, v_{4}\right)$ denote the quadrilateral with the vertices $v_{1}, v_{2}, v_{3}, v_{4}$ and with the generic edge-lines $\left(v_{1}, v_{2}\right),\left(v_{2}, v_{3}\right),\left(v_{3}, v_{4}\right),\left(v_{4}, v_{1}\right)$. The lines $\left(v_{1}, v_{3}\right)$ and $\left(v_{2}, v_{4}\right)$ are the diagonals of the quadrilateral. The notation $p_{i, i+1}$ will be used to denote a point that is contained in the line $\left(v_{i}, v_{i+1}\right)$. Proposition 2.1 is a degenerate case of Brianchon's theorem [2,13].

Proposition 2.1 Let $p_{1,2}, p_{2,3}, p_{3,4}, p_{4,1}$ be the four tangency points of a nondegenerate conic that is inscribed in a quadrilateral in $\mathbb{P}^{2}$. Then, the lines $\left(p_{1,2}, p_{3,4}\right)$ and $\left(p_{2,3}, p_{4,1}\right)$ are concurrent with the two diagonals of the quadrilateral. (See Fig. 4.)

Let $p_{1,2}, p_{2,3}, p_{3,1}$ be points that are distinct from the vertices of a triangle $\triangle\left(v_{1}, v_{2}, v_{3}\right)$. The points form a Ceva configuration if and only if the three lines $\left(v_{1}, p_{2,3}\right),\left(v_{2}, p_{3,1}\right)$, and $\left(v_{3}, p_{1,2}\right)$ are concurrent. The points form a Menelaus configuration if and only if the three points $p_{1,2}, p_{2,3}$, and $p_{3,1}$ are collinear. (See Fig. 2.)

Theorem 2.2 (Ceva's and Menelaus' theorems) Consider a triangle $\triangle\left(v_{1}, v_{2}, v_{3}\right)$ in the affine plane. Let $p_{1,2}, p_{2,3}$, and $p_{3,1}$ be points on the respective edge-lines $\left(v_{1}, v_{2}\right)$, $\left(v_{2}, v_{3}\right)$, and $\left(v_{3}, v_{1}\right)$ that are distinct from the vertices of the triangle. Then,

$$
\frac{l\left(v_{1}, p_{1,2}\right)}{l\left(p_{1,2}, v_{2}\right)} \cdot \frac{l\left(v_{2}, p_{2,3}\right)}{l\left(p_{2,3}, v_{3}\right)} \cdot \frac{l\left(v_{3}, p_{3,1}\right)}{l\left(p_{3,1}, v_{1}\right)}
$$

(i) equals 1 if and only if the points form a Ceva configuration, 

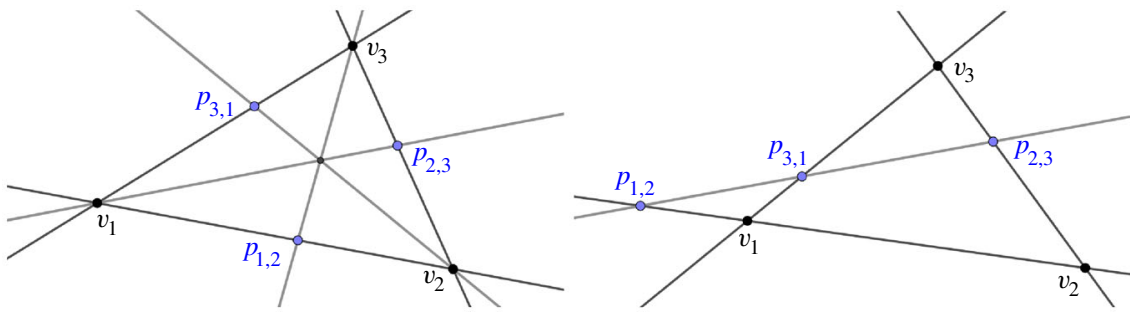

Fig. 2 For Ceva configurations and also for Menelaus configurations, the placement of the point $p_{3,1}$ can be uniquely determined by the placement of the points $p_{1,2}$ and $p_{2,3}$

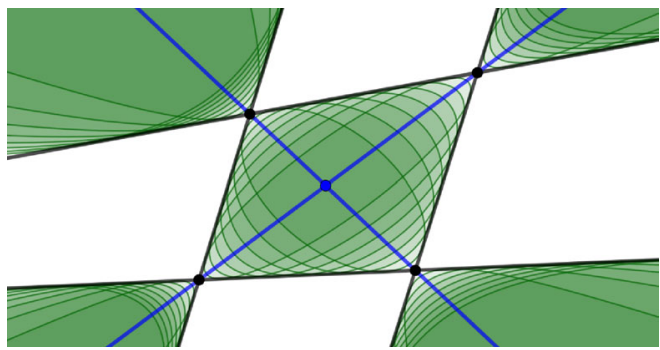

Fig. 3 For any quadrilateral, there is a 1-parameter family of inscribed conics. The three diagonals of the complete quadrilateral are degenerate inscribed conics

(ii) equals -1 if and only if the points form a Menelaus configuration.

Here, $l(v, p)$ denotes an oriented length.

Note that the quotient of the oriented lengths is invariant with respect to the line orientation. Theorem 2.2 can be found, for example, in [6,19,20].

Proposition 2.3 is a limit of the well-known fact that there is a unique non-degenerate conic tangent to five generic lines. A proof can be found in [8], for example.

Proposition 2.3 For any quadrilateral in $\mathbb{P}^{2}$, there exists a 1-parameter family of inscribed conics. (See Fig. 3.) Any non-degenerate inscribed conic can be uniquely determined by specifying one of its tangency points that is not a vertex of the complete quadrilateral.

Theorem 2.4 Let $p_{1,2}, p_{2,3}, p_{3,4}, p_{4,1}$ be four distinct points that are distinct from the vertices of a quadrilateral $\square\left(v_{1}, v_{2}, v_{3}, v_{4}\right)$ in $\mathbb{P}^{2}$. These points determine a Ceva configuration for each of the triangles $\triangle\left(v_{1}, v_{2}, v_{3}\right), \triangle\left(v_{2}, v_{3}, v_{4}\right), \triangle\left(v_{3}, v_{4}, v_{1}\right)$, $\triangle\left(v_{4}, v_{1}, v_{2}\right)$. The intersection point of the diagonals of $\square\left(v_{1}, v_{2}, v_{3}, v_{4}\right)$ is a common point of the four Ceva configurations if and only if the points $p_{1,2}, p_{2,3}, p_{3,4}, p_{4,1}$ are the tangency points of a non-degenerate inscribed conic. (See Fig. 4.)

Proof By applying a projective transformation to $\mathbb{P}^{2}$, the quadrilateral $\square\left(v_{1}, v_{2}, v_{3}, v_{4}\right)$ can be mapped to a square. Then, any inscribed conic is symmetric with respect to the diagonals of the square. So, the intersection point of the diagonals is a common point of the four Ceva configurations. For the converse, suppose that the intersection 


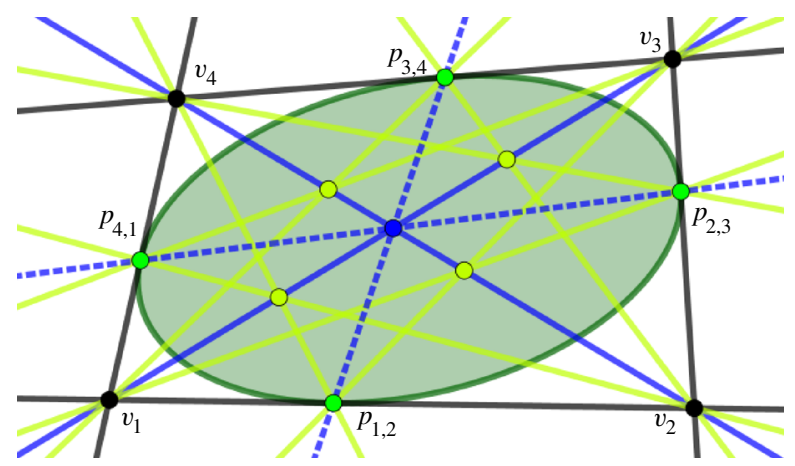

Fig. 4 For any non-degenerate conic that is inscribed in the quadrilateral, the two lines connecting the opposite tangency points are concurrent with the two diagonals. The tangency points determine a Ceva configuration on each of the triangles $\Delta\left(v_{1}, v_{2}, v_{3}\right), \Delta\left(v_{2}, v_{3}, v_{4}\right), \Delta\left(v_{3}, v_{4}, v_{1}\right), \Delta\left(v_{4}, v_{1}, v_{2}\right)$. The intersection point $\left(v_{1}, v_{3}\right) \cap\left(v_{2}, v_{4}\right)$ is a common point of the four Ceva configurations

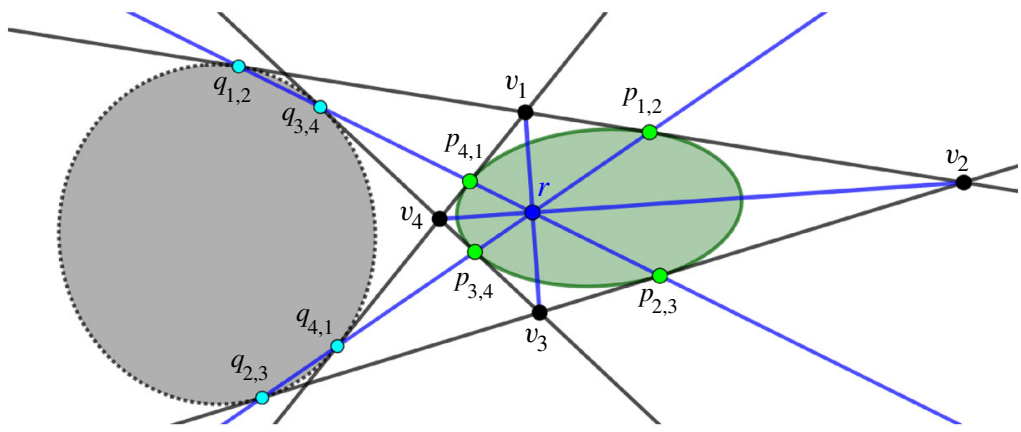

Fig. 5 The points $p_{1,2}, p_{2,3}, p_{3,4}, p_{4,1}$ are the tangency points of an inscribed conic. Lemma 2.5 defines an inscribed conic with tangency points $q_{1,2}, q_{2,3}, q_{3,4}, q_{4,1}$ such that $q_{1,2}, q_{3,4}, p_{2,3}, p_{4,1}$ are collinear and $q_{2,3}, q_{4,1}, p_{1,2}, p_{3,4}$ are collinear

point of the diagonals is a common point of the four Ceva configurations. Then, the four points on the edge-lines of the square must be symmetric about the diagonals of the square. So, they are the tangency points of an inscribed conic.

Theorem 2.4 is a generalisation of the fact that Ceva configurations correspond to non-degenerate conics that are inscribed in triangles.

Lemma 2.5 Consider a quadrilateral $\square\left(v_{1}, v_{2}, v_{3}, v_{4}\right)$ in $\mathbb{P}^{2}$ and let $r$ be the intersection point of the diagonals. Let $p_{1,2}, p_{2,3}, p_{3,4}, p_{4,1}$ be the tangency points of an inscribed conic $\mathscr{C}$. As shown in Fig. 5, construct the points $q_{1,2}, q_{2,3}, q_{3,4}, q_{4,1}$ by drawing the two lines containing the collinear points $\left\{p_{1,2}, r, p_{3,4}\right\}$ and $\left\{p_{2,3}, r, p_{4,1}\right\}$. The points $q_{1,2}, q_{2,3}, q_{3,4}, q_{4,1}$ are the tangency points of a conic that is inscribed in $\square\left(v_{1}, v_{2}, v_{3}, v_{4}\right)$.

Proof By applying a projective transformation, the quadrilateral $\square\left(v_{1}, v_{2}, v_{3}, v_{4}\right)$ can be mapped to a square. Then, the inscribed conic $\mathscr{C}$ is symmetric with respect to the two diagonals of the quadrilateral. The two lines containing the collinear points 

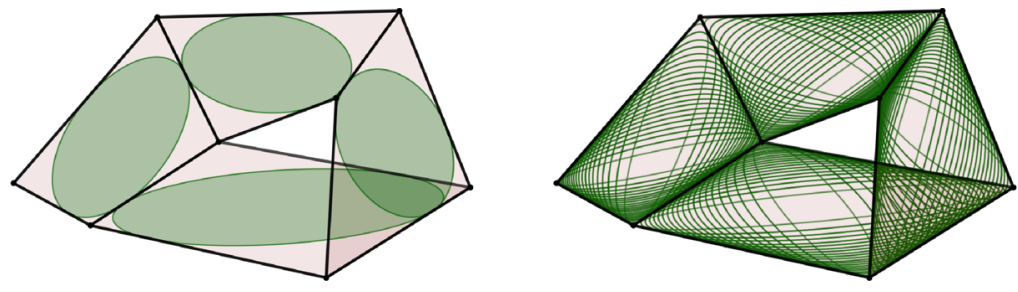

Fig. 6 If a bipartite loop of planar quadrilaterals admits one instance of touching inscribed conics, then it admits a 1-parameter family of touching inscribed conics

$\left\{p_{1,2}, r, p_{3,4}\right\}$ and $\left\{p_{2,3}, r, p_{4,1}\right\}$ are also symmetric with respect to both of the diagonals. In particular, the points $q_{1,2}, q_{2,3}, q_{3,4}, q_{4,1}$ are symmetric with respect to both of the diagonals. So, by Theorem 2.4, they are the tangency points of an inscribed conic.

For any quadrilateral $\square\left(v_{1}, v_{2}, v_{3}, v_{4}\right)$ in $\mathbb{P}^{2}$, Lemma 2.5 establishes an involution on the 1-parameter family of inscribed conics. However, there is one degenerate case. For any quadrilateral, there is a unique inscribed conic that is projectively equivalent to a circle inscribed in a square. It is mapped under the involution to a degenerate inscribed conic, namely the double line $\left(\left(v_{1}, v_{2}\right) \cap\left(v_{3}, v_{4}\right),\left(v_{2}, v_{3}\right) \cap\left(v_{4}, v_{1}\right)\right)$. We are mostly interested in the generic case.

\section{Nets of Planar Quadrilaterals with Touching Inscribed Conics}

\subsection{Porism}

In projective space $\mathbb{P}^{n}, n \geq 2$, nets of planar quadrilaterals (or Q-nets) are discrete surfaces that are defined by gluing together planar quadrilaterals. By definition, two planar quadrilaterals are glued together if and only if they have two common vertices on a common edge-line. Nets of planar quadrilaterals with touching inscribed conics are nets of planar quadrilaterals such that each planar quadrilateral is equipped with an inscribed conic such that, for any two neighbouring quadrilaterals, the two inscribed conics have the same tangency point on their common edge-line.

A loop of planar quadrilaterals is a net of planar quadrilaterals where every quadrilateral is glued with exactly two other quadrilaterals. A loop of planar quadrilaterals is called bipartite if the vertices can be bicoloured so that the vertices have different colours if they share an edge.

Theorem 3.1 Consider a bipartite loop of finitely many planar quadrilaterals in projective space $\mathbb{P}^{n}, n \geq 2$. If it admits one instance of touching inscribed conics, then it admits a 1-parameter family of touching inscribed conics (See Fig. 6.)

Proof Enumerate the quadrilaterals $\left\{Q_{i}\right\}_{i=1}^{n}$ of the bipartite loop so that $Q_{i}$ and $Q_{i+1}$ are neighbouring quadrilaterals for any $i \in \mathbb{Z} / n \mathbb{Z}$. Let $l_{i}$ denote the common edge-line of the two neighbouring quadrilaterals $Q_{i}$ and $Q_{i+1}$. Let $r_{i}$ denote the intersection of 

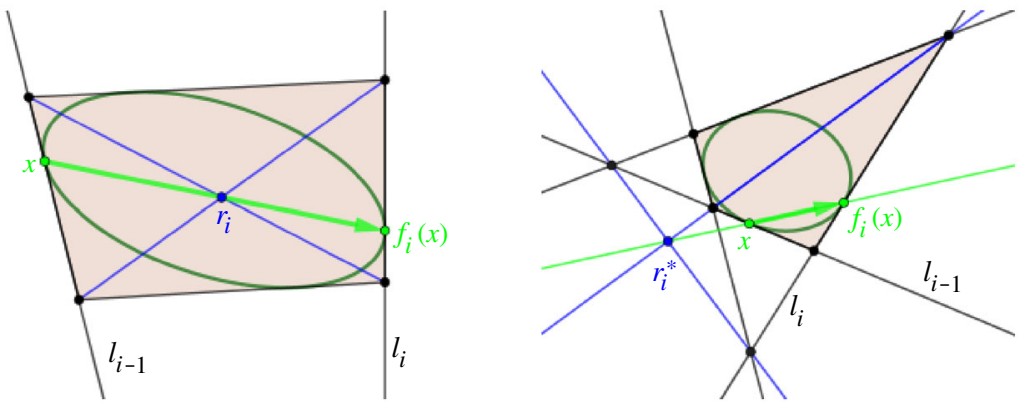

Fig. 7 The central projection $f_{i}: l_{i-1} \rightarrow l_{i}$ is defined in the proof of Theorem 3.1. In the first case, the centre is $r_{i}$. In the second case, the centre is $r_{i}^{*}$

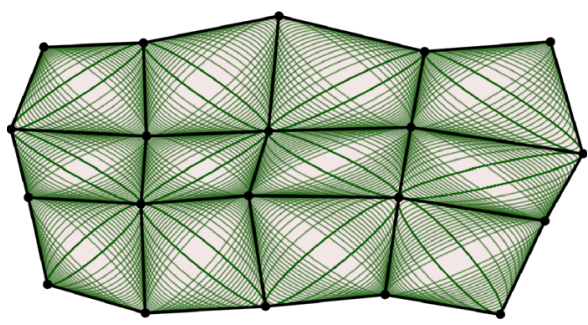

Fig. 8 If a Q-net admits an instance of touching inscribed conics, then it admits a 1-parameter family of touching inscribed conics and, by Theorem 3.5, it is a Koenigs net

the diagonals of $Q_{i}$. For each $i \in \mathbb{Z} / n \mathbb{Z}$, define a central projection $f_{i}: l_{i-1} \rightarrow l_{i}$. There are two cases to consider. First, suppose that the lines $l_{i-1}$ and $l_{i}$ do not intersect at a vertex of the quadrilateral $Q_{i}$, as shown in the left of Fig. 7. Then $f_{i}$ is defined to be the central projection with centre $r_{i}$. Second, suppose that the lines $l_{i-1}$ and $l_{i}$ do intersect at a vertex of the quadrilateral $Q_{i}$, as shown in the right of Fig. 7. Then, the map $f_{i}: l_{i-1} \rightarrow l_{i}$ is defined to be the central projection with centre $r_{i}^{*}$ defined as the intersection of two of the three diagonals of the complete quadrilateral determined by $Q_{i}$. The two diagonals are chosen so that they determine a quadrilateral where $l_{i-1}$ and $l_{i}$ are opposite edge-lines. Because the loop is bipartite, the two common vertices of the quadrilaterals $Q_{n}$ and $Q_{1}$ are two fixed points of the projective transformation $f:=f_{n} \circ f_{n-1} \circ \cdots \circ f_{2} \circ f_{1}: l_{0} \rightarrow l_{0}$. Suppose that the bipartite loop admits an instance of touching inscribed conics. For each conic inscribed in $Q_{i}$, Proposition 2.1 shows that the central projection $f_{i}$ maps the tangency point of $l_{i-1}$ to the tangency point of $l_{i}$. So, the touching point on the line $l_{0}$ is a fixed point of the projective transformation $f$. The map $f$ must be the identity because it has three fixed points. So the loop admits a 1-parameter family of touching inscribed conics.

Corollary 3.2 A Q-net $f: \mathbb{Z}^{2} \rightarrow \mathbb{P}^{n}, n \geq 2$, admits an instance of touching inscribed conics if and only if it admits a 1-parameter family of touching inscribed conics. (See Fig. 8.) 
Theorem 3.3 In projective space $\mathbb{P}^{n}, n \geq 2$, the double cover of a non-bipartite loop always admits a 1-parameter family of touching inscribed conics.

Proof As in the proof of Theorem 3.1, enumerate the quadrilaterals and let $l_{i}$ denote the common edge-line of the two neighbouring quadrilaterals $Q_{i}$ and $Q_{i+1}$ for all $i \in \mathbb{Z} / n \mathbb{Z}$. Define $f: l_{0} \rightarrow l_{0}$ to be the projective transformation $f_{n} \circ f_{n-1} \circ \cdots \circ f_{2} \circ f_{1}$ that was defined in the proof of Theorem 3.1. Let $v_{1}$ and $v_{2}$ be the two common vertices of $Q_{1}$ and $Q_{n}$. Because the loop of planar quadrilaterals is not bipartite, it follows that $f\left(v_{1}\right)=v_{2}$ and $f\left(v_{2}\right)=v_{1}$. However, any projective transformation $\mathbb{P}^{1} \rightarrow \mathbb{P}^{1}$ is an involution if it exchanges two distinct points [20, Lem. 8.1]. Therefore, $f \circ f \equiv \mathrm{id}$.

\subsection{Koenigs Nets}

Two planar quadrilaterals $\square(a, b, c, d)$ and $\square\left(a^{*}, b^{*}, c^{*}, d^{*}\right)$ in $\mathbb{R}^{n}, n \geq 2$, are called dual quadrilaterals if and only if their corresponding edge-lines are parallel and their non-corresponding diagonals are parallel. For any planar quadrilateral, a dual quadrilateral exists and it is uniquely determined up to translation and rescaling. It is worth mentioning that the notion of dual quadrilaterals is not projective.

A net $f: \mathbb{Z}^{2} \rightarrow \mathbb{A}^{n}$ of planar quadrilaterals in affine space $\mathbb{A}^{n}, n \geq 2$, is called a 2-dimensional Koenigs net if there exists a Christoffel dual net $f^{*}: \overline{\mathbb{Z}}^{2} \rightarrow \mathbb{A}^{n}$ such that the corresponding quadrilaterals are dual [6]. Although 2-dimensional Koenigs nets are defined in terms of affine geometry, it is known that the class of 2-dimensional Koenigs nets is invariant under projective transformations.

Definition 3.4 Consider a Q-net $f: \mathbb{Z}^{2} \rightarrow \mathbb{A}^{n}, n \geq 2$. Denote by $M_{i, j}$ the intersection point of the diagonals of the quadrilateral $\square\left(f_{i, j}, f_{i+1, j}, f_{i+1, j+1}, f_{i, j+1}\right)$. Then, the net $f: \mathbb{Z}^{2} \rightarrow \mathbb{A}^{n}$ is a 2-dimensional Koenigs net if and only if the following condition is satisfied for all $(i, j) \in \mathbb{Z}^{2}$ :

$$
\frac{l\left(M_{i, j}, f_{i+1, j}\right)}{l\left(M_{i, j}, f_{i, j+1}\right)} \cdot \frac{l\left(M_{i-1, j}, f_{i, j+1}\right)}{l\left(M_{i-1, j}, f_{i-1, j}\right)} \cdot \frac{l\left(M_{i-1, j-1}, f_{i-1, j}\right)}{l\left(M_{i-1, j-1}, f_{i, j-1}\right)} \cdot \frac{l\left(M_{i, j-1}, f_{i, j-1}\right)}{l\left(M_{i, j-1}, f_{i+1, j}\right)}=1
$$

This algebraic characterisation and further projective geometric properties of Koenigs nets can be found in [6].

Theorem 3.5 A Q-net $f: \mathbb{Z}^{2} \rightarrow \mathbb{P}^{n}, n \geq 2$, is a 2-dimensional Koenigs net if and only if it admits (a 1-parameter family of) touching inscribed conics.

Proof Consider the four quadrilaterals of the Q-net in Fig. 9. Suppose that the four quadrilaterals have touching inscribed conics. Equivalently, by Theorem 2.4, the touching points of the inscribed conics determine four Ceva configurations that are highlighted in Fig. 9. The points $M$, which are the intersections of the diagonals of the quadrilaterals, are points of the four Ceva configurations. Each of the Ceva configurations implies the multi-ratio identity (i) of Theorem 2.2, saying that the product of three ratios of oriented lengths equals 1 . Consider the product of the four equations. The product gives the identity (1) because the lengths can be oriented so that cancellations 


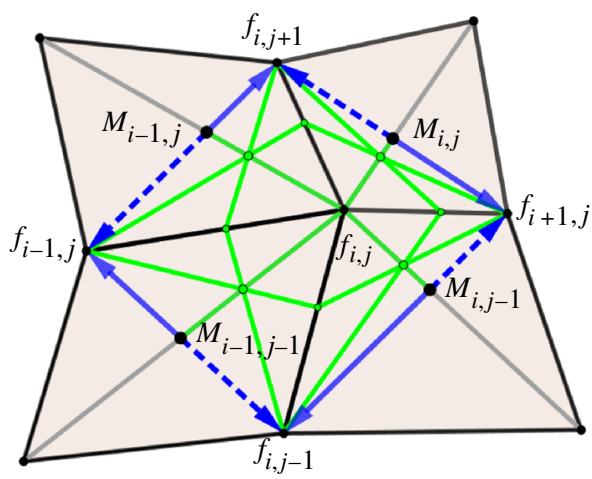

Fig. 9 The identity (1) is valid if and only if the net of planar quadrilaterals admits a 1-parameter family of touching inscribed conics. The identity (1) says that the product of the oriented lengths of the dashed arrows is equal to the product of the oriented lengths of the solid arrows

occur on the common edge-lines of the four Ceva configurations. For the converse, Ceva's theorem can be used to show that the identity (1) is sufficient to ensure that there is a 1-parameter family of Ceva configurations as shown in Fig. 9. Equivalently, by Theorem 2.4, there is a 1-parameter family of touching inscribed conics.

Corollary 3.6 If all the edge-lines of a $Q$-net $f: \mathbb{Z}^{2} \rightarrow \mathbb{P}^{n}, n \geq 2$, are tangent to a quadric and if none of the planes of the $Q$-net are contained in the quadric, then generically $f$ is a 2-dimensional Koenigs net.

Proof Consider a planar quadrilateral of the Q-net. As the plane of the quadrilateral is not contained in the quadric, the plane intersects the quadric in a conic. Generically, the conic is non-degenerate. So, the Q-net admits an instance of touching inscribed conics. By Theorem 3.5, the Q-net is a Koenigs net.

Examples of Q-nets with their edge-lines tangent to a sphere are given by Koebe polyhedra, which are used in [3] to construct discrete minimal surfaces. The corresponding touching conics are circles. Koebe polyhedra have a 1-parameter family of touching inscribed conics.

\section{Line Grids with Quadrilaterals with Touching Inscribed Conics}

\subsection{Polygonal Chains Inscribed in Conics}

Now we are going to give a direct application of Lemma 2.5. Let $p_{0}, p_{1}, \ldots, p_{m}$ and $q_{0}, q_{1}, \ldots, q_{n}$ be the vertices of two polygonal chains that are inscribed in a non-degenerate conic $\mathscr{C}$. Let $k_{0}, k_{1}, \ldots, k_{m}$ and $l_{0}, l_{1}, \ldots, l_{n}$ be the respective tangent lines of $\mathscr{C}$. The notation $\square\left(k_{i-1}, l_{j-1}, k_{i}, l_{j}\right)$ stands for the quadrilateral with the vertices $k_{i-1} \cap l_{j-1}, k_{i-1} \cap l_{j}, k_{i} \cap l_{j}, k_{i} \cap l_{j-1}$. By Lemma 2.5, the points $\left(p_{i-1}, p_{i}\right) \cap l_{j-1},\left(p_{i-1}, p_{i}\right) \cap l_{j},\left(q_{j-1}, q_{j}\right) \cap k_{i-1},\left(q_{j-1}, q_{j}\right) \cap k_{i}$ are the tangency points of a conic that is inscribed in $\square\left(k_{i-1}, l_{j-1}, k_{i}, l_{j}\right)$. Therefore, the $m \times n$ 


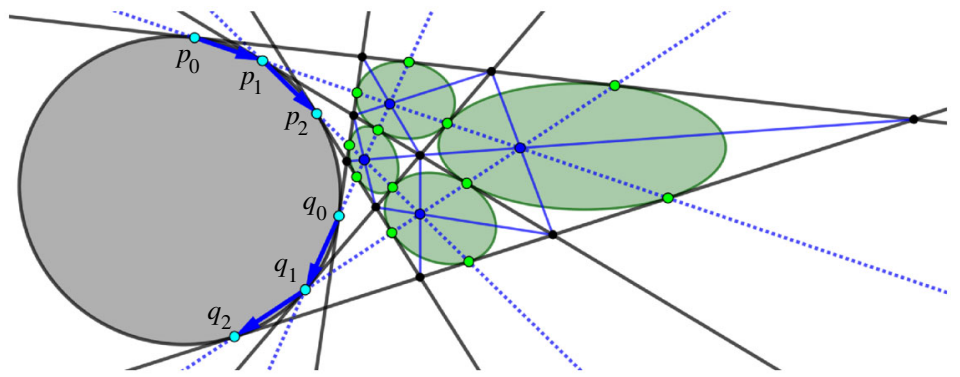

Fig. 10 Two polygonal chains are inscribed in a conic. The dotted lines are the lines $\left(p_{0}, p_{1}\right),\left(p_{1}, p_{2}\right),\left(q_{0}, q_{1}\right),\left(q_{1}, q_{2}\right)$. By construction, the tangency points of the touching inscribed conics lie on the dotted lines

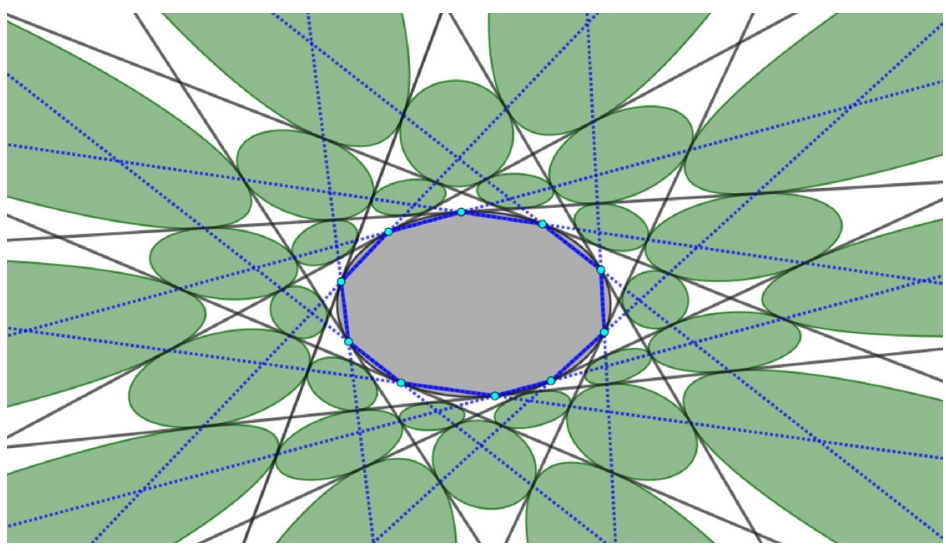

Fig. 11 An inscribed polygon and a grid of quadrilaterals with touching inscribed conics such that the tangency points are contained in the dotted edge-lines of the inscribed polygon

grid $\left\{\square\left(k_{i-1}, l_{j-1}, k_{i}, l_{j}\right)\right\}_{1 \leq i \leq m, 1 \leq j \leq n}$ admits an instance of touching inscribed conics such that the tangency points satisfy some non-trivial collinearities. An example is shown in Fig. 10 where the non-trivial collinearities are represented by the dotted lines.

In the above construction of grids of quadrilaterals with touching inscribed conics, the two polygonal chains determine the "horizontal" and "vertical" lines of the grid. However, they can be merged. (See Fig. 11.)

\subsection{Generic Lines Tangent to a Conic}

Theorem 4.1 is a consequence of two classical theorems which are referenced in the proof.

Theorem 4.1 Let $k_{0}, k_{1}$ and $l_{0}, l_{1}, \ldots, l_{n}, n \geq 2$, be lines in the projective plane such that each quadrilateral $Q_{i}:=\square\left(k_{0}, l_{i-1}, k_{1}, l_{i}\right)$ has four generic edge-lines. Let $r_{i}$ be the intersection point of the diagonals of $Q_{i}$. Then the following are equivalent: 
(i) The lines $k_{0}, k_{1}$ and $l_{0}, l_{1}, \ldots, l_{n}$ are tangent to a non-degenerate conic.

(ii) The points $\left\{r_{i}\right\}_{i=1}^{n}$ lie in a line that does not contain the point $k_{0} \cap k_{1}$.

Suppose that the lines $k_{0}, k_{1}$ and $l_{0}, l_{1}, \ldots, l_{n}$ are tangent to a non-degenerate conic $\mathscr{C}$. Let $k_{0,1}$ be the line containing the collinear points $\left\{r_{i}\right\}_{i=1}^{n}$. Then, $k_{0} \cap k_{0,1}$ and $k_{1} \cap k_{0,1}$ are the tangency points of the tangent lines $k_{0}$ and $k_{1}$.

Proof By the dual of Steiner's theorem on the projective generation of non-degenerate conics, the lines $k_{0}, k_{1}, l_{0}, l_{1}, \ldots, l_{n}$ are tangent to a non-degenerate conic if and only if there is a projective transformation $f: k_{0} \rightarrow k_{1}$ such that $f\left(k_{0} \cap l_{i}\right)=k_{1} \cap l_{i}$ for all $i \in$ $\{1, \ldots, n\}$ and such that $f\left(k_{0} \cap k_{1}\right) \neq k_{0} \cap k_{1}$ [8, Thms. 8.1.4 and 8.1.8]. Equivalently, by the cross-axis theorem [8, Thm. 5.3.5] and [8, Prop. 5.3.7], the points $\left\{r_{i}\right\}_{i=1}^{n}$ are contained in a line which is called the cross-axis of $f: k_{0} \rightarrow k_{1}$. The cross-axis is not concurrent with the lines $k_{0}$ and $k_{1}$, because otherwise the projective transformation $f: k_{0} \rightarrow k_{1}$ would be a central projection so that $f\left(k_{0} \cap k_{1}\right)=k_{0} \cap k_{1}$. Therefore (i) and (ii) are equivalent. Suppose that the generic lines $k_{0}, k_{1}$ and $l_{0}, l_{1}, \ldots, l_{n}$ are tangent to a non-degenerate conic $\mathscr{C}$. Let $p_{0}$ and $p_{1}$ be the tangency points of the tangent lines $k_{0}$ and $k_{1}$. Because $\mathscr{C}$ is inscribed in each of the quadrilaterals $\left\{Q_{i}\right\}_{i=1}^{n}$, Proposition 2.1 ensures that the points $\left\{r_{i}\right\}_{i=1}^{n}$ are contained in the line $\left(p_{0}, p_{1}\right)$. Therefore, $p_{0}=k_{0} \cap k_{0,1}$ and $p_{1}=k_{1} \cap k_{0,1}$.

Let $k_{0}, k_{1}, \ldots, k_{m}$ and $l_{0}, l_{1}, \ldots, l_{n}$ be generic lines in the projective plane. Consider the $m \times n$ grid of quadrilaterals $Q_{i, j}:=\square\left(k_{i-1}, l_{j-1}, k_{i}, l_{j}\right)$. We use $K_{i-1, i}$ and $L_{j-1, j}$ to denote the strips of quadrilaterals $\left\{\square\left(k_{i-1}, l_{j-1}, k_{i}, l_{j}\right)\right\}_{j=1}^{n}$ and $\left\{\square\left(k_{i-1}, l_{j-1}, k_{i}, l_{j}\right)\right\}_{i=1}^{m}$, respectively.

Theorem 4.2 For six generic lines $k_{0}, k_{1}, k_{2}, l_{0}, l_{1}, l_{2}$ in the projective plane, consider the $2 \times 2$ grid of quadrilaterals $Q_{i, j}:=\square\left(k_{i-1}, l_{j-1}, k_{i}, l_{j}\right)$. We use $r_{i, j}$ to denote the intersection point of the diagonals of the quadrilateral $Q_{i, j}$. Then the following are equivalent:

(i) The six lines $k_{0}, k_{1}, k_{2}, l_{0}, l_{1}, l_{1}$ are tangent to a non-degenerate conic.

(ii) The $2 \times 2$ grid of quadrilaterals admits an instance of touching inscribed conics $\mathscr{C}_{i, j}$ such that the following sets are sets of collinear points. (See Fig. 12.)

$$
\begin{array}{ll}
\left\{K_{0,1} l_{0}, r_{1,1}, K_{0,1} l_{1}, r_{1,2}, K_{0,1} l_{2}\right\}, & \left\{k_{0} L_{1,2}, r_{1,2}, k_{1} L_{1,2}, r_{2,2}, k_{2} L_{1,2}\right\}, \\
\left\{K_{1,2} l_{0}, r_{2,1}, K_{1,2} l_{1}, r_{2,2}, K_{1,2} l_{2}\right\}, & \left\{k_{0} L_{0,1}, r_{1,1}, k_{1} L_{0,1}, r_{2,1}, k_{2} L_{0,1}\right\} .
\end{array}
$$

The points $K_{i-1, i} l_{j-1}, K_{i-1, i} l_{j}, k_{i-1} L_{j-1, j}, k_{i} L_{j-1, j}$ are defined to be the tangency points of the conic $\mathscr{C}_{i, j}$ that is inscribed in the quadrilateral $Q_{i, j}$. The tangency points are labelled by their tangent lines and by the strips of quadrilaterals.

(iii) The $2 \times 2$ grid of quadrilaterals admits an instance of touching inscribed conics.

(iv) The $2 \times 2$ grid of quadrilaterals admits a 1-parameter family of touching inscribed conics.

(v) The three lines $\left(r_{1,1}, r_{2,1}\right),\left(r_{1,2}, r_{2,2}\right)$, and $l_{1}$ are concurrent.

(vi) The three lines $\left(r_{1,1}, r_{1,2}\right),\left(r_{2,1}, r_{2,2}\right)$, and $k_{1}$ are concurrent. 


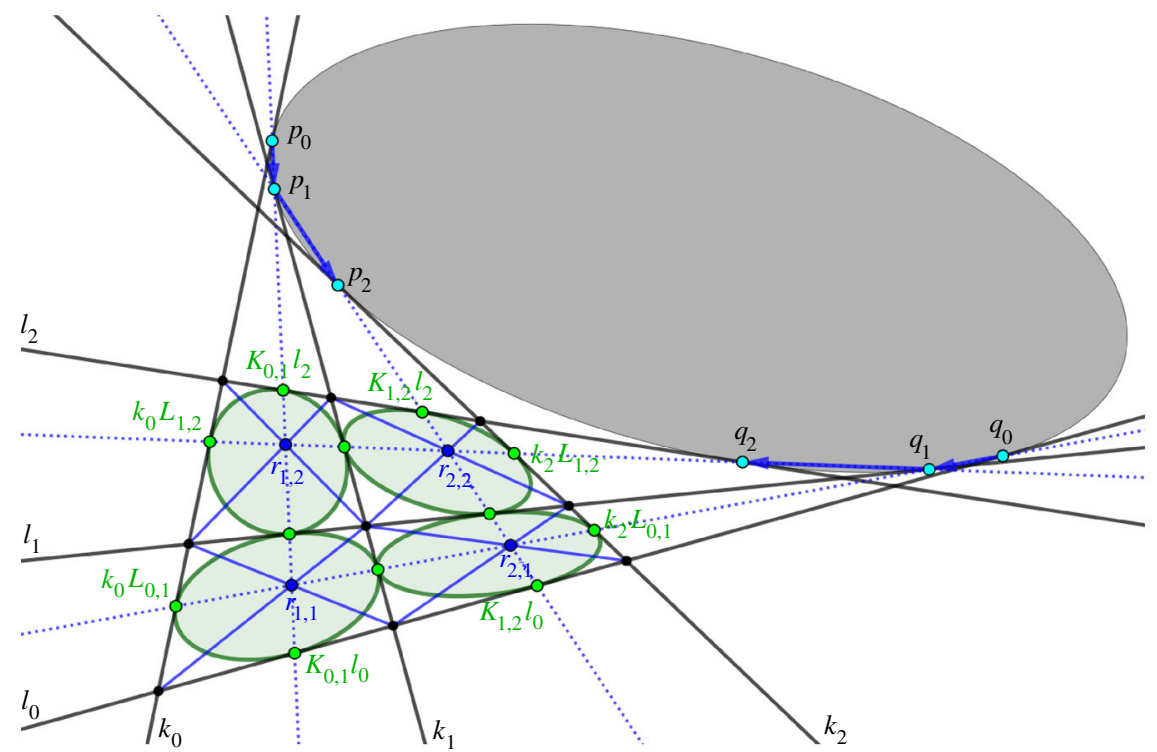

Fig. 12 The six generic gridlines are tangent to a non-degenerate conic if and only if the $2 \times 2$ grid of quadrilaterals admits a 1-parameter family of touching inscribed conics. Among the 1-parameter family of touching inscribed conics, there is one instance of touching inscribed conics such that the tangency points satisfy some non-trivial collinearities which are represented by the dotted lines

Proof Suppose that the generic lines $k_{0}, k_{1}, k_{2}, l_{0}, l_{1}, l_{2}$ are tangent to a non-degenerate conic $\mathscr{C}$. Let $p_{0}, p_{1}, p_{2}, q_{0}, q_{1}, q_{2}$ be the tangency points of the tangent lines $k_{0}, k_{1}, k_{2}, l_{0}, l_{1}, l_{2}$. Consider the two polygonal chains $p_{0}, p_{1}, p_{2}$ and $q_{0}, q_{1}, q_{2}$ that are inscribed in the non-degenerate conic $\mathscr{C}$. By the construction in Sect. 4.1, the $2 \times 2$ grid of quadrilaterals admits an instance of touching inscribed conics such that the following sets are sets of collinear points:

$$
\begin{array}{ll}
\left\{K_{0,1} l_{0}, r_{1,1}, K_{0,1} l_{1}, r_{1,2}, K_{0,1} l_{2}\right\}, & \left\{k_{0} L_{1,2}, r_{1,2}, k_{1} L_{1,2}, r_{2,2}, k_{2} L_{1,2}\right\}, \\
\left\{K_{1,2} l_{0}, r_{2,1}, K_{1,2} l_{1}, r_{2,2}, K_{1,2} l_{2}\right\}, & \left\{k_{0} L_{0,1}, r_{1,1}, k_{1} L_{0,1}, r_{2,1}, k_{2} L_{0,1}\right\} .
\end{array}
$$

Therefore, (i) implies (ii). Obviously, (ii) implies (iii). By Theorem 3.1, (iii) implies (iv).

Suppose that the $2 \times 2$ grid of quadrilaterals admits a 1-parameter family of touching inscribed conics. By Theorem 3.5, it is a Koenigs net. So, in any affine image of $\mathbb{P}^{2}$,

$$
\frac{l\left(k_{0} \cap l_{1}, r_{1,1}\right)}{l\left(r_{1,1}, k_{1} \cap l_{0}\right)} \cdot \frac{l\left(k_{1} \cap l_{0}, r_{2,1}\right)}{l\left(r_{2,1}, k_{2} \cap l_{1}\right)} \cdot \frac{l\left(k_{2} \cap l_{1}, r_{2,2}\right)}{l\left(r_{2,2}, k_{1} \cap l_{2}\right)} \cdot \frac{l\left(k_{1} \cap l_{2}, r_{1,2}\right)}{l\left(r_{1,2}, k_{0} \cap l_{1}\right)}=1 .
$$

By applying Menelaus' theorem to the triangles $\Delta\left(k_{0} \cap l_{1}, k_{2} \cap l_{1}, k_{1} \cap l_{2}\right)$ and $\triangle\left(k_{0} \cap\right.$ $\left.l_{1}, k_{2} \cap l_{1}, k_{1} \cap l_{0}\right)$, the identity (2) implies that the two lines $\left(r_{1,1}, r_{2,1}\right)$ and $\left(r_{1,2}, r_{2,2}\right)$ are concurrent with the line $l_{1}$. By applying Menelaus' theorem to the triangles $\triangle\left(k_{1} \cap\right.$ $\left.l_{0}, k_{1} \cap l_{2}, k_{0} \cap l_{1}\right)$ and $\triangle\left(k_{1} \cap l_{0}, k_{1} \cap l_{2}, k_{2} \cap l_{1}\right)$, the identity (2) implies that the two 
lines $\left(r_{1,1}, r_{1,2}\right)$ and $\left(r_{2,1}, r_{2,2}\right)$ are concurrent with the line $k_{1}$. Therefore, (iv) implies both (v) and (vi).

Suppose that the three lines $\left(r_{1,1}, r_{2,1}\right),\left(r_{1,2}, r_{2,2}\right)$, and $l_{1}$ are concurrent. Let $q_{1}$ be the concurrency point. The generic lines $k_{0}, k_{1}, k_{2}, l_{0}, l_{1}$ are tangent to a uniquely determined non-degenerate conic [8, Cor. 8.1.12], say $\mathscr{A}$. By Theorem 4.1, $q_{1}$ is a tangency point of $\mathscr{A}$. Likewise, the generic lines $k_{0}, k_{1}, k_{2}, l_{1}, l_{2}$ are tangent to a uniquely determined non-degenerate conic, say $\mathscr{B}$, with the tangency point $q_{1}$. Then, by Proposition $2.3, \mathscr{A}=\mathscr{B}$ because $\mathscr{A}$ and $\mathscr{B}$ have four common generic tangent lines $k_{0}, k_{1}, k_{2}, l_{1}$ and the common tangency point $q_{1}$. Therefore, (v) implies (i). Symmetrically, (vi) also implies (i).

Corollary 4.3 Let $k_{0}, k_{1}, \ldots, k_{m}, m \geq 2$, and $l_{0}, l_{1}, \ldots, l_{n}, n \geq 3$, be generic lines in the projective plane. Consider the $m \times n$ grid of quadrilaterals $Q_{i, j}:=$ $\square\left(k_{i-1}, l_{j-1}, k_{i}, l_{j}\right)$. We use $r_{i, j}$ to denote the intersection point of the diagonals of the quadrilateral $Q_{i, j}$. Then the following are equivalent:

(i) The generic lines $k_{0}, k_{1}, \ldots, k_{m}, l_{0}, l_{1}, \ldots, l_{n}$ are tangent to a non-degenerate conic.

(ii) The $m \times n$ grid of quadrilaterals admits an instance of touching inscribed conics $\mathscr{C}_{i, j}$ such that the following are collections of sets of collinear points. (See Fig. 13.)

$$
\left\{\left\{K_{i-1, i} l_{j}\right\}_{j=0}^{n}\right\}_{i=1}^{m}, \quad\left\{\left\{k_{i} L_{j-1, j}\right\}_{i=0}^{m}\right\}_{j=1}^{n} \text {. }
$$

The points $K_{i-1, i} l_{j-1}, K_{i-1, i} l_{j}, k_{i-1} L_{j-1, j}, k_{i} L_{j-1, j}$ are defined to be the tangency points of the conic $\mathscr{C}_{i, j}$ that is inscribed in the quadrilateral $Q_{i, j}$. The tangency points are labelled by their tangent lines and by the strips of quadrilaterals.

(iii) The $m \times n$ grid of quadrilaterals admits an instance of touching inscribed conics.

(iv) The $m \times n$ grid of quadrilaterals admits a 1-parameter family of touching inscribed conics.

(v) $\left\{\left\{r_{i, j}\right\}_{i=1}^{m}\right\}_{j=1}^{n}$ and $\left\{\left\{r_{i, j}\right\}_{j=1}^{n}\right\}_{i=1}^{m}$ are collections of sets of collinear points.

(vi) $\left\{\left\{r_{i, j}\right\}_{j=1}^{n}\right\}_{i=1}^{m}$ is a collection of sets of collinear points.

Proof As in the proof of Theorem 4.2, (i) $\Rightarrow$ (ii) $\Rightarrow$ (iii) $\Rightarrow$ (iv) $\Rightarrow$ (v) $\Rightarrow$ (vi) are straightforward. The only step we comment on is (vi) $\Rightarrow(\mathrm{i})$. Suppose that $\left\{\left\{r_{i, j}\right\}_{j=1}^{n}\right\}_{i=1}^{m}$ is a collection of sets of collinear points. By Theorem 4.1, for any $i \in\{1, \ldots, m\}$, the generic lines $k_{i-1}, k_{i}, l_{0}, l_{1}, \ldots, l_{n}$ are tangent to a non-degenerate conic, say $\mathscr{C}_{i}$. For any $i \in\{1, \ldots, m-1\}$, the non-degenerate conics $\mathscr{C}_{i}$ and $\mathscr{C}_{i+1}$ are identical because they have five common tangent lines $k_{i}, l_{0}, l_{1}, l_{2}, l_{3}$. Therefore, (vi) $\Rightarrow$ (i).

By Corollary 4.3, any generic net of lines with the combinatorics of the square grid and with all elementary quadrilaterals possessing touching inscribed conics can be constructed from two polygonal chains that are inscribed in a non-degenerate conic as explained in Sect. 4.1. The inscribed conics of two combinatorially neighbouring quadrilaterals have the same touching point on their common edge-line. Corollary 4.3 implies the incidence theorem that is illustrated in Fig. 14 

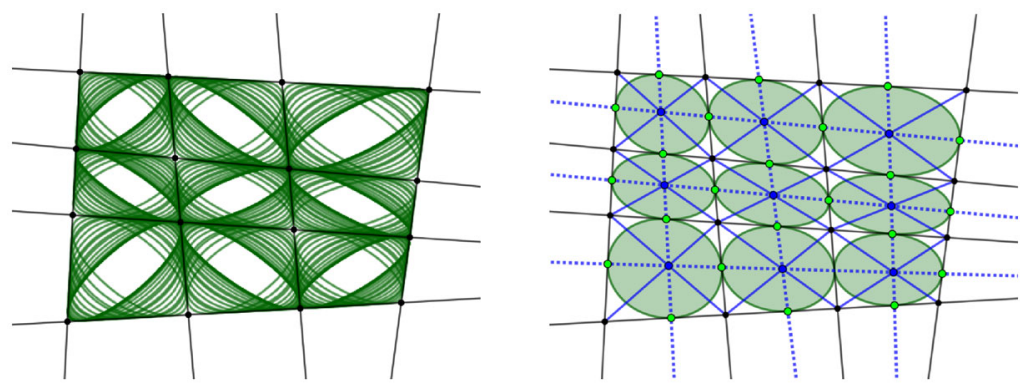

Fig. 13 If a $3 \times 3$ grid of quadrilaterals admits an instance of touching inscribed conics, then there is a 1-parameter family of touching inscribed conics. Among the 1-parameter family, there is one instance of touching inscribed conics such that the tangency points satisfy some non-trivial collinearities which are represented by the dotted lines

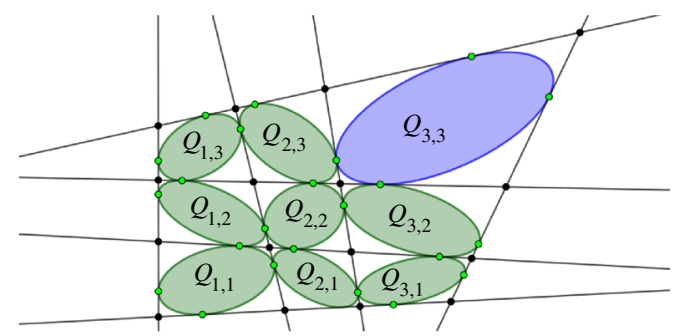

Fig. 14 Suppose that each of the quadrilaterals $Q_{1,1}, Q_{1,2}, Q_{1,3}, Q_{2,1}, Q_{2,2}, Q_{2,3}, Q_{3,1}, Q_{3,2}$ is equipped with an inscribed conic such that, for any two neighbouring quadrilaterals, the inscribed conics are touching. Then, $Q_{3,3}$ admits an inscribed conic that touches the two conics that are inscribed in $Q_{3,2}$ and $Q_{2,3}$. By Corollary 4.3 , the eight lines are tangent to a conic

Koenigs nets can be treated as discrete conjugate nets with equal Laplace invariants [6]. By Theorem 3.5, grids of quadrilaterals with touching inscribed conics are planar 2-dimensional Koenigs nets. It is worth mentioning that Koenigs showed in [16] that planar nets with equal Laplace invariants can be understood locally by the condition that six lines are tangent to a conic.

\subsection{Conics Associated to the Strips}

Theorem 4.4 Let $k_{0}, k_{1}, \ldots, k_{m}$ and $l_{0}, l_{1}, \ldots, l_{n}, m, n \in \mathbb{N}_{\geq 2}$, be generic lines in the projective plane. Suppose that the $m \times n$ grid of quadrilaterals $Q_{i, j}:=$ $\square\left(k_{i-1}, l_{j-1}, k_{i}, l_{j}\right)$ has touching inscribed conics. Equivalently, the gridlines are tangent to a conic $\mathscr{C}$. Then, along each strip of quadrilaterals, the touching points are contained in a conic that has double contact with $\mathscr{C}$. (See Fig. 15.)

We start with Lemma 4.5, which will be used in the proof of Theorem 4.4.

Lemma 4.5 Let $p_{1,2}, p_{2,3}, p_{3,4}, p_{4,1}$ be the tangency points of a conic that is inscribed in a quadrilateral $\square\left(v_{1}, v_{2}, v_{3}, v_{4}\right)$ in $\mathbb{P}^{2}$. Let $q_{1,2}, q_{2,3}, q_{3,4}, q_{4,1}$ be the tangency points of another inscribed conic. Then, there exists a unique conic containing the 


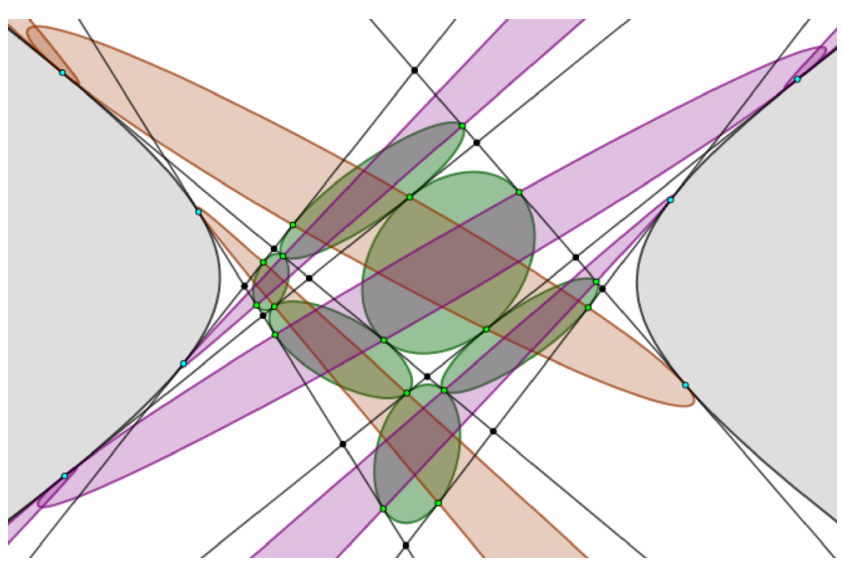

Fig. 15 The gridlines are tangent to a conic $\mathscr{C}$. The tangency points of the touching inscribed conics are contained in conics that have double contact with $\mathscr{C}$

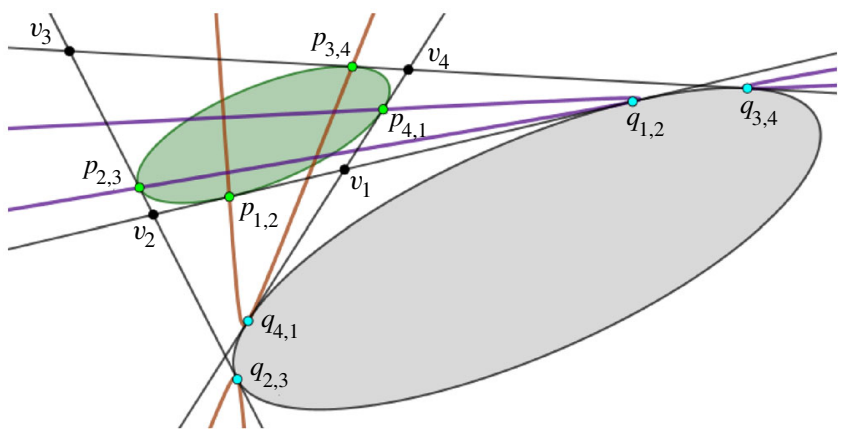

Fig. 16 For any two inscribed conics, there exists a conic containing the points $p_{1,2}, q_{2,3}, p_{3,4}, q_{4,1}$ and with the tangent lines $\left(v_{2}, v_{3}\right),\left(v_{4}, v_{1}\right)$. Symmetrically, there exists a conic containing the points $q_{1,2}, p_{2,3}, q_{3,4}, p_{4,1}$ and with the tangent lines $\left(v_{1}, v_{2}\right),\left(v_{3}, v_{4}\right)$

points $p_{1,2}, q_{2,3}, p_{3,4}, q_{4,1}$ and tangent to the lines $\left(v_{2}, v_{3}\right)$ and $\left(v_{4}, v_{1}\right)$. Symmetrically, there exists a unique conic containing the points $q_{1,2}, p_{2,3}, q_{3,4}, p_{4,1}$ and with the tangent lines $\left(v_{1}, v_{2}\right)$ and $\left(v_{3}, v_{4}\right)$. (See Fig. 16.)

Proof By applying a projective transformation, the quadrilateral $\square\left(v_{1}, v_{2}, v_{3}, v_{4}\right)$ can be mapped to a square. Let $r$ be the intersection point of the diagonals of the square. Consider the pencil of conics through the four points $p_{1,2}, q_{2,3}, p_{3,4}, q_{4,1}$. Any conic in the pencil can be determined by the four base points and a fifth point. One can show, for example by using Pascal's theorem, that the reflection about $r$ of the fifth point is also contained in the conic. So, all of the conics in the pencil are symmetric about the point $r$. Therefore, if a conic containing the points $p_{1,2}, q_{2,3}, p_{3,4}, q_{4,1}$ is tangent to the line $\left(v_{2}, v_{3}\right)$, then it must also be tangent to the line $\left(v_{4}, v_{1}\right)$ because the two lines are symmetric about the point $r$.

Proof of Theorem 4.4 The quadrilaterals $Q_{i, j}$ are equipped with inscribed conics $\mathscr{C}_{i, j}$ such that, for any two neighbouring quadrilaterals, the inscribed conics are touching. 


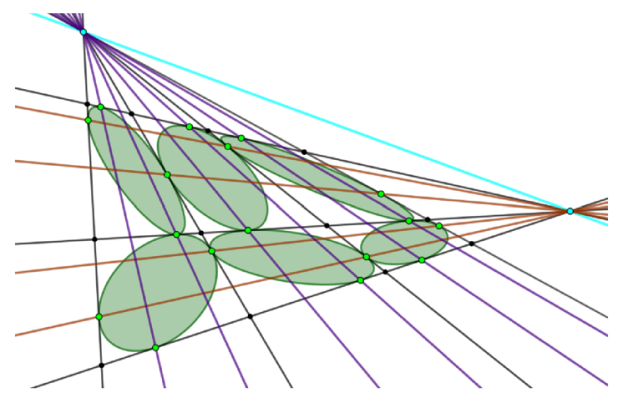

Fig. 17 A non-generic $3 \times 2$ grid of quadrilaterals with touching inscribed conics. The corresponding common conic is degenerate. The lines $k_{i}$ are concurrent and so too are the lines $l_{j}$. For each strip of quadrilaterals, the double contact conics containing the tangency points are also degenerate. They are pairs of lines passing through the concurrency points

Let $k_{i-1} L_{j-1, j}, k_{i} L_{j-1, j}, K_{i-1, i} l_{j-1}, K_{i-1, i} l_{j}$ be the corresponding tangency points, labelled by their tangent lines and by the strips of quadrilaterals. By Corollary 4.3, the lines $k_{0}, k_{1}, \ldots, k_{m}$ and $l_{0}, l_{1}, \ldots, l_{n}$ are tangent to a non-degenerate conic $\mathscr{C}$. Let $p_{0}, p_{1}, \ldots, p_{m}$ and $q_{0}, q_{1}, \ldots, q_{n}$ be the respective tangency points. The aim is to show that for any fixed $i$, the points $\left\{K_{i-1, i} l_{j}\right\}_{j=0}^{n}$ are contained in a conic $\mathscr{A}_{i}$ that has double contact with the conic $\mathscr{C}$. Symmetrically, for any fixed $j$, the aim is to show that the points $\left\{k_{i} L_{j-1, j}\right\}_{i=0}^{m}$ are contained in a conic $\mathscr{B}_{j}$ that has double contact with the conic $\mathscr{C}$. For any fixed $i \in\{1, \ldots, m\}$, consider the strip $K_{i-1, i}$ of quadrilaterals $\left\{Q_{i, j}\right\}_{j=1}^{n}$. For each quadrilateral $Q_{i, j}$ in $K_{i-1, i}$, Lemma 4.5 determines a conic, say $\mathscr{D}_{j}$, containing the points $p_{i-1}, p_{i}, K_{i-1, i} l_{j-1}, K_{i-1, i} l_{j}$ and with the tangent lines $k_{i-1}, k_{i}$. The conics $\mathscr{D}_{j}$ and $\mathscr{D}_{j+1}$ are equal because $p_{i-1}, p_{i}, K_{i-1, i} l_{j}$ are common points and the lines $k_{i-1}, k_{i}$ are common tangents. Thus, $\mathscr{A}_{i}$ is the conic $\mathscr{D}_{1}=\ldots=\mathscr{D}_{n}$. Therefore, the conics $\left\{\mathscr{A}_{i}\right\}_{i=1}^{m}$ exist and, symmetrically, the conics $\left\{\mathscr{B}_{j}\right\}_{j=1}^{n}$ also exist. Figure 17 illustrates a degenerate case of Theorem 4.4.

\subsection{Incircular Nets and Billiards in Conics}

Incircular nets are line grids with quadrilaterals with inscribed circles. The following characterisation of incircular nets can be found in [1, Defn. 2.3].

Definition 4.6 Let $a_{0}, a_{1}, \ldots, a_{m}$ and $b_{0}, b_{1}, \ldots, b_{n}$ be lines in the Euclidean plane. The $m \times n$ grid of quadrilaterals $\square\left(a_{i-1}, b_{j-1}, a_{i}, b_{j}\right)$ is an incircular net if and only if the following conditions are satisfied.

(i) The lines $a_{0}, a_{1}, \ldots, a_{m}$ and $b_{0}, \ldots, b_{n}$ are tangent to a conic $\mathscr{C}$.

(ii) The points $a_{i-1} \cap a_{i}$ and $b_{j-1} \cap b_{j}$ are contained in a conic $\mathscr{D}$ that is confocal with $\mathscr{C}$.

Billiards in conics are examples of integrable systems. An important property of billiards in conics is that they have caustics that are confocal conics. (See Fig. 18.) An introduction to billiards can be found in [22]. The lines $a_{0}, a_{1}, \ldots, a_{m}$ and $b_{0}, b_{1}, \ldots, b_{n}$ in Definition 4.6 can be interpreted as the lines of two billiards in 


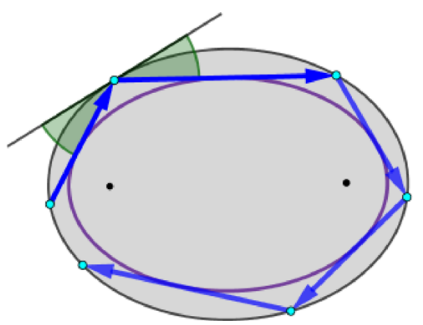

Fig. 18 The billiard reflection law says that the angle of reflection equals the angle of incidence. For billiards in conics, the trajectory forever remains tangent to a confocal conic

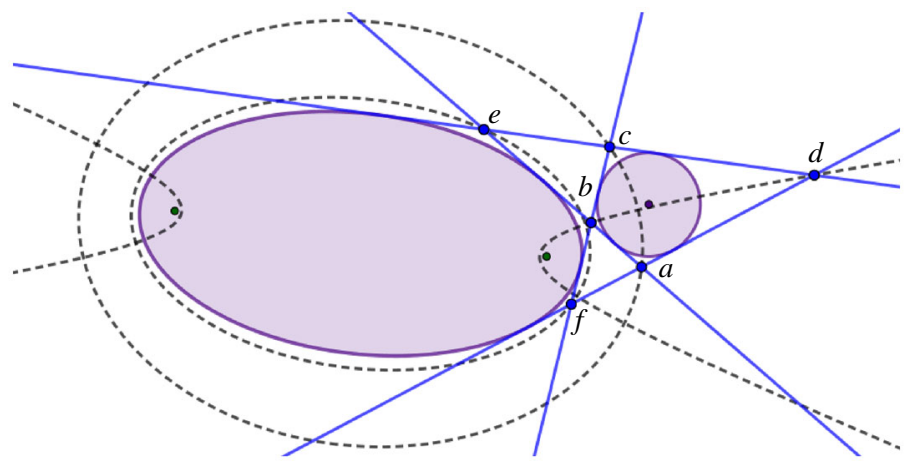

Fig. 19 The complete quadrilateral has an inscribed circle if and only if, for any inscribed conic, there are three confocal conics that contain the pairs of opposite vertices

the conic $\mathscr{D}$ that have the same confocal caustic $\mathscr{C}$. Confocal conics have four common imaginary tangent lines [18]. Conics that are tangent to four real lines appeared in the study of elliptic billiards in the Lorentz plane [15].

The gridlines of any incircular net are tangent to a conic. Therefore, by Corollary 4.3, incircular nets are grids of quadrilaterals that admit a 1-parameter family of touching inscribed conics. However, for two neighbouring quadrilaterals of an incircular net, the two inscribed circles are generically not touching as they do not have the same tangency point on the common edge-line of the two quadrilaterals. (See Fig. 1.)

Theorem 4.7 For any incircular net, there is a dual grid of quadrilaterals that has a 1-parameter family of touching inscribed conics. The vertices of the dual grid are the centres of the circles of the incircular net. The lines of the dual grid are angle bisector lines of the incircular net. (See Fig. 1.)

The proof of Theorem 4.7 uses the Graves-Chasles theorem, a proof of which can be found in [1,11], for example. See also [14].

Theorem 4.8 (Graves-Chasles Theorem) Suppose that a conic $\mathscr{C}$ is tangent to the four edge-lines of a complete quadrilateral in $\mathbb{R}^{2}$. Denote the pairs of opposite vertices by $\{a, c\},\{b, d\},\{e, f\}$ as shown in Fig. 19. Then the following are equivalent:

(i) There exists a circle that is tangent to the four edge-lines. 


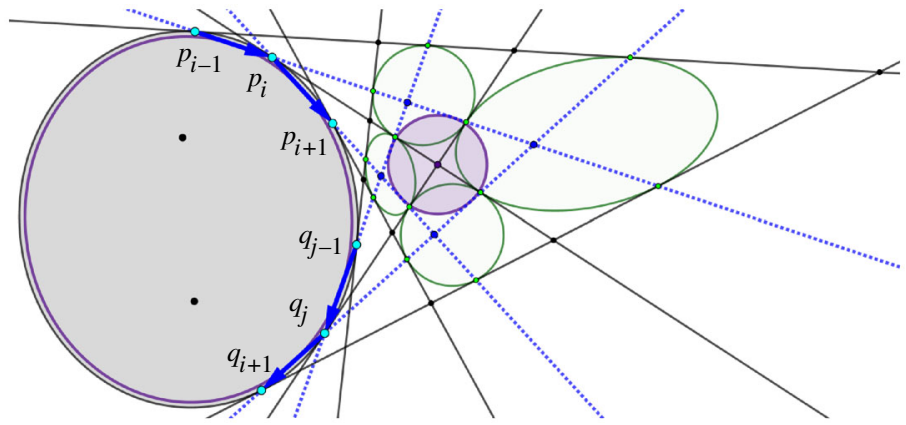

Fig. 20 The two polygonal chains $p_{i-1}, p_{i}, p_{i+1}$ and $q_{j-1}, q_{j}, q_{j+1}$ are billiards that are inscribed in a conic $\mathscr{D}$. Suppose that the two billiards have the same confocal caustic. Then, by the Graves-Chasles theorem, there exists a circle that is tangent to the four dotted lines. The centre of the circle is the intersection point of the tangent lines of $\mathscr{D}$ at $p_{i}$ and $q_{j}$. These tangent lines generate a line grid with touching inscribed conics

(ii) The points $\{a, c\}$ lie on a conic that is confocal with $\mathscr{C}$.

(iii) The points $\{b, d\}$ lie on a conic that is confocal with $\mathscr{C}$.

(iv) The points $\{e, f\}$ lie on a conic that is confocal with $\mathscr{C}$.

If the inscribed circle exists, then the tangent lines of the confocal conics at the vertices of the complete quadrilateral are concurrent at the centre of the inscribed circle.

Proof of Theorem 4.7 Any incircular net determines two billiards $p_{0}, p_{1}, \ldots, p_{m}$ and $q_{0}, q_{1}, \ldots, q_{n}$ that are inscribed in a conic $\mathscr{D}$ and that have the same confocal caustic $\mathscr{C}$. Let $k_{0}, k_{1}, \ldots, k_{m}$ and $l_{0}, l_{1}, \ldots, l_{n}$ be the tangent lines of $\mathscr{D}$ at the points $p_{0}, p_{1}, \ldots, p_{m}$ and $q_{0}, q_{1}, \ldots, q_{n}$, respectively. By Corollary 4.3, the $m \times n$ grid of quadrilaterals $\square\left(k_{i-1}, l_{j-1}, k_{i}, l_{j}\right)$ admits a 1 -parameter family of touching inscribed conics. The billiard reflection law ensures that the tangent line of $\mathscr{D}$ at $p_{i}$ is an angle bisector of the lines $\left(p_{i-1}, p_{i}\right)$ and $\left(p_{i}, p_{i+1}\right)$. In other words, the tangent line of $\mathscr{D}$ at $p_{i}$ divides one of the angles between the two lines $\left(p_{i-1}, p_{i}\right)$ and $\left(p_{i}, p_{i+1}\right)$ in half. Likewise, the tangent line of $\mathscr{D}$ at $q_{j}$ is an angle bisector of the lines $\left(q_{j-1}, q_{j}\right)$ and $\left(q_{j}, q_{j+1}\right)$. By Theorem 4.8 , there is a circle that is tangent to the four lines $\left(p_{i-1}, p_{i}\right),\left(p_{i}, p_{i+1}\right),\left(q_{j-1}, q_{j}\right),\left(q_{j}, q_{j+1}\right)$ and that is centred at the intersection point of the tangent lines of $\mathscr{D}$ at $p_{i}$ and $q_{j}$. (See Fig. 20.)

Acknowledgements Thanks to the anonymous referees for valuable suggestions that improved the paper.

Funding Open Access funding enabled and organized by Projekt DEAL.

Open Access This article is licensed under a Creative Commons Attribution 4.0 International License, which permits use, sharing, adaptation, distribution and reproduction in any medium or format, as long as you give appropriate credit to the original author(s) and the source, provide a link to the Creative Commons licence, and indicate if changes were made. The images or other third party material in this article are included in the article's Creative Commons licence, unless indicated otherwise in a credit line to the material. If material is not included in the article's Creative Commons licence and your intended use is not permitted by statutory regulation or exceeds the permitted use, you will need to obtain permission directly from the copyright holder. To view a copy of this licence, visit http://creativecommons.org/licenses/by/4.0/. 


\section{References}

1. Akopyan, A.V., Bobenko, A.I.: Incircular nets and confocal conics. Trans. Am. Math. Soc. 370(4), 2825-2854 (2018)

2. Berger, M.: Geometry II. Universitext. Springer, Berlin (2009)

3. Bobenko, A.I., Hoffmann, T., Springborn, B.A.: Minimal surfaces from circle patterns: geometry from combinatorics. Ann. Math. 164(1), 231-264 (2006)

4. Bobenko, A.I., Lutz, C.O.R., Pottmann, H., Techter, J.: Non-Euclidean Laguerre geometry and incircular nets (2020). arXiv:2009.00978

5. Bobenko, A.I., Schief, W.K., Techter, J.: Checkerboard incircular nets: Laguerre geometry and parametrisation. Geom. Dedicata 204, 97-129 (2020)

6. Bobenko, A.I., Suris, Yu.B.: Discrete Differential Geometry. Integrable Structure. Graduate Studies in Mathematics, vol. 98. American Mathematical Society, Providence (2008)

7. Böhm, W.: Verwandte Sätze über Kreisvierseitnetze. Arch. Math. (Basel) 21, 326-330 (1970)

8. Casas-Alvero, E.: Analytic Projective Geometry. EMS Textbooks in Mathematics. European Mathematical Society, Zürich (2014)

9. Chasles, M.: Traité des Sections Coniques Faisant Suite au Traité de Géométrie Supérieure. Premiere Partie. Thesaurus Mathematicae, vol. 3. Physica, Würzburg (1962)

10. Darboux, G.: Lecons sur la Théorie Générale des Surfaces et les Applications Géométriques du Calcul Infinitésimal, vol. 2 \& 3. Gauthier-Villars \& Fils, Paris $(1887,1889)$

11. Darboux, G.: Principes de Géométrie Analytique. Gauthier-Villars, Paris (1917)

12. Dragović, V., Radnović, M.: Poncelet Porisms and Beyond. Integrable Billiards, Hyperelliptic Jacobians and Pencils of Quadrics. Frontiers in Mathematics. Birkhäuser, Basel (2011)

13. Glaeser, G., Stachel, H., Odehnal, B.: The Universe of Conics. Springer Spektrum, Berlin (2016)

14. Izmestiev, I., Tabachnikov, S.: Ivory's theorem revisited. J. Integrable Syst. 2(1), \# xyx006 (2017)

15. Khesin, B., Tabachnikov, S.: Pseudo-Riemannian geodesics and billiards. Adv. Math. 221(4), 13641396 (2009)

16. Kœnigs, G.: Sur les reseaux plans à invariants égaux et les lignes asymptotiques. Comptes Rendus Acad. Sci. 114, 55-57 (1892)

17. Levi, M., Tabachnikov, S.: The Poncelet grid and billiards in ellipses. Am. Math. Mon. 114(10), 895-908 (2007)

18. Poncelet, J.-V.: Traité des Propriétés Projectives des Figures, vol. 1. Gauthier-Villars, Paris (1865)

19. Richter-Gebert, J.: Meditations on Ceva's theorem. In: The Coxeter Legacy-Reflections and Projections (Toronto 2004), pp. 227-254. American Mathematical Society, Providence (2006)

20. Richter-Gebert, J.: Perspectives on Projective Geometry. A Guided Tour Through Real and Complex Geometry. Springer, Heidelberg (2011)

21. Schwartz, R.E.: The Poncelet grid. Adv. Geom. 7(2), 157-175 (2007)

22. Tabachnikov, S.: Geometry and Billiards. Student Mathematical Library, vol. 30. American Mathematical Society, Providence (2005)

Publisher's Note Springer Nature remains neutral with regard to jurisdictional claims in published maps and institutional affiliations. 